\title{
Normal Shock Wave-Turbulent Boundary Layer interactions in transonic intakes at incidence
}

\author{
A. Coschignano* and H. Babinsky ${ }^{\dagger}$ \\ Department of Engineering, University of Cambridge, Cambridge, CB2 1PZ, UK \\ C. Sheaf $f^{\ddagger}$ and E. Platt $\ddagger$ \\ Installation Aerodynamics, Rolls Royce Plc., Derby, DE24 8BJ, UK
}

The flow field around a transonic engine inlet lip at high incidence is investigated for a variety of flow conditions around the design point. Generally, the flow on the upper surface of the lip is characterised by a supersonic region, terminated by a near-normal shock wave. At the nominal design point, the shock is not strong enough to cause significant flow separation, resulting only in marginal losses in pressure recovery. Off-design conditions were explored by altering the angle of attack as well as changing the mass flow rate over the upper lip, intended to mimic the effect of an increase in engine flow. The results suggest that angle of attack has the greatest effect on the flow field. In particular, even a relatively small increase of $2^{\circ}$ can lead to large and highly unsteady flow separation with an associated shock oscillation. Both qualitative and quantitative measurements suggest a noticeably reduced aerodynamic performance resulting from higher incidence operation. In contrast, an increase of up to $5.2 \%$ in mass flow over the upper part of the intake lip did not result in large separated regions or flow-field unsteadiness.

\section{Nomenclature}

$\alpha \quad$ Angle of incidence

$\delta \quad$ Boundary layer thickness

$\delta^{*} \quad$ Displacement thickness

$\theta \quad$ momentum thickness

$A(T)$ Pressure sensitive calibration constant

$B(T)$ Pressure sensitive calibration constant

$c \quad$ Intake chord length

$H$ Shape factor

$I_{\%} \quad$ Free-stream turbulence

I Pressure sensitive paint luminescence

$\dot{m}$ Mass flow

$M \quad$ Mach number

$P \quad$ Pressure

Re Reynolds number (based on lip thickness)

$s \quad$ Stream-wise distance along surface

$t_{m} \quad$ Intake maximum thickness

$U \quad$ Flow velocity

$w \quad$ Wind tunnel width

$x \quad$ Stream-wise direction, parallel to lab floor

y Vertical direction, normal to surface model, unless otherwise stated

$z \quad$ Span-wise direction.

${ }^{*}$ PhD Candidate, Department of Engineering, University of Cambridge, AIAA Student Member.

$\dagger$ Professor of Aerodynamics, Department of Engineering, University of Cambridge, AIAA Associate Fellow.

${ }^{\ddagger}$ Installation Aerodynamics, Rolls Royce Plc, Derby. 
LDV Laser Doppler velocimetry

PSP Pressure sensitive paint

RMS Root mean square

SBLI Shock-wave boundary layer interaction

Subscript

$0 \quad$ Stagnation value

1 Property upstream of the shock

e $\quad$ Free-stream property

$l \quad$ lower channel, usually referred to mass flows

$i \quad$ Incompressible property

$u \quad$ upper channel, usually referred to mass flows

\section{Shock-Boundary Layer Interactions in Subsonic Engine Intakes}

When operating under high-thrust conditions, such as during take-off and climb, the substantial mass flow demand by a turbofan engine is sufficient to accelerate the flow over the intake lip to supersonic conditions. This faster-than sound flow pocket is terminated by a near-normal shock wave. This is schematically depicted in Figure 1. The adverse pressure gradient imposed by this disturbance on the boundary layer can cause the latter to separate, introducing large scale unsteadiness and an increase in viscous losses 1 These losses have a direct negative repercussion on the overall engine efficiency and operability as the total pressure reaching the fan face is reduced. Moreover, if the separated boundary layer does not reattach before the fan face, the unsteadiness, characteristic of separated flows, may increase the stress on the fan, which can ultimately reduce component lives and aerodynamic stability margin.

Although a significant amount of research has gone into reducing the detrimental effects associated with shock-induced separation in transonic flight, the majority of these efforts have been limited to aerofoil and wing flows. The formation of shock waves on the inside lip of engine intakes has often been overlooked and, so far, only highlight (nacelle leading edge) separation at large incidence $\left(>30^{\circ}\right)$ has been investigated in detail $2 \sqrt[3]{3}$ However, these early experiments were characterised by poor resolution and limited measurements. The onset of separation near the throat, which precedes highlight stall, ${ }^{2}$ has not been considered. Therefore, not only is there an insufficient understanding surrounding the onset of separation, but also these experiments might have overestimated the capabilities of the intake to provide clean flow at milder incidence levels. Furthermore, the lack of high quality experimental data has hindered the development of accurate CFD codes to assess this phenomena. To address this shortcoming, Cambridge University has started experimental research into this problem. The first stage of this project investigated the flow field occurring during typical take-off or climb conditions and found the shock-boundary interaction to be relatively benign $4 \sqrt{5}$ Separated flow was found to be confined to a small region of space. However, aero-engine intakes are designed to operate over a wide range, defined by inflow speed, incidence and engine demand. The consequences of increasing the last two parameters are considered in the current investigation. In particular, the main interest is to assess the onset and severity of any unsteady regimes.

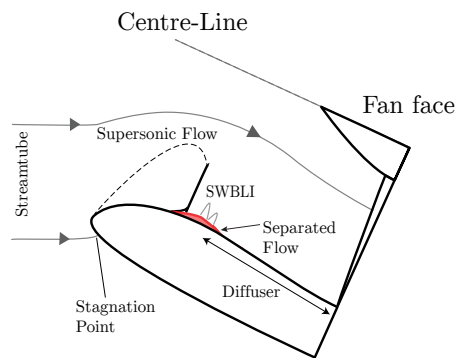

On-design

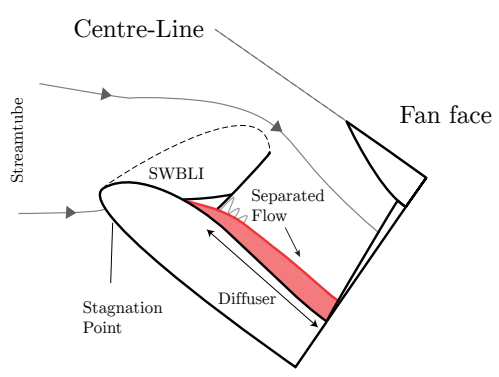

Off-design

Figure 1. Schematic representation of two flow scenarios characterised by different degrees of shock-induced boundary layer separation over an intake lip cross-section during high-incidence flight. 


\section{Experimental set-up and methodology}

\section{A. Experimental facility}

All experiments are performed in the high-speed aerodynamics laboratory at the University of Cambridge. In particular, a blow-down wind tunnel, powered by two $50 \mathrm{~kW}$ compressors, is used. The flow is fed from the compressors into the settling chamber, where it is passed through a number of flow straighteners and turbulence grids before a 18:1 contraction. In the current configuration, no nozzle is used and the entry velocity is varied by changing the stagnation pressure and the effective area of the second throat where the flow is chocked. By altering the cross-sectional area of the second throat by means of an aerofoil (see Figure 2 the overall mass flow rate is adjusted, controlling the entry Mach number with a resolution of \pm 0.001 . The unique working section was designed by Makun ${ }^{\sqrt{5}}$ exclusively for the investigation of shock wave boundary layer interactions in engine intakes and it is depicted in Figure 2. This features curved upper and lower walls, forming a stream-tube divided by a $1 / 7^{\text {th }}$ scale intake lip model and provides a way to replicate the confinement imposed onto the flow-field by the engine spinner. The stream-tube geometry is based on 3D computational results performed for a realistic intake. ${ }^{5}$ The upper boundary is a streamline of the computed flow far enough from the supersonic region to avoid choking in the upper channel. The highest supply pressure possible is 2.4 bar. ${ }^{5}$ The experimental operating range is portrayed in Figure 3 . The rig is capable of achieving entry conditions of $\mathrm{M}_{\text {entry }}=0.25 \rightarrow 0.45$. The characteristic length used for calculating the Reynolds number is the maximum intake lip thickness. In the Mach number range considered, the experiment is capable of matching full scale Reynolds numbers for sea level and 5000m for a typical small and large engine respectively. Evidence suggests no Reynolds dependence within this range and a nominal Re of approximately 1.23 million is used (Figure 3).

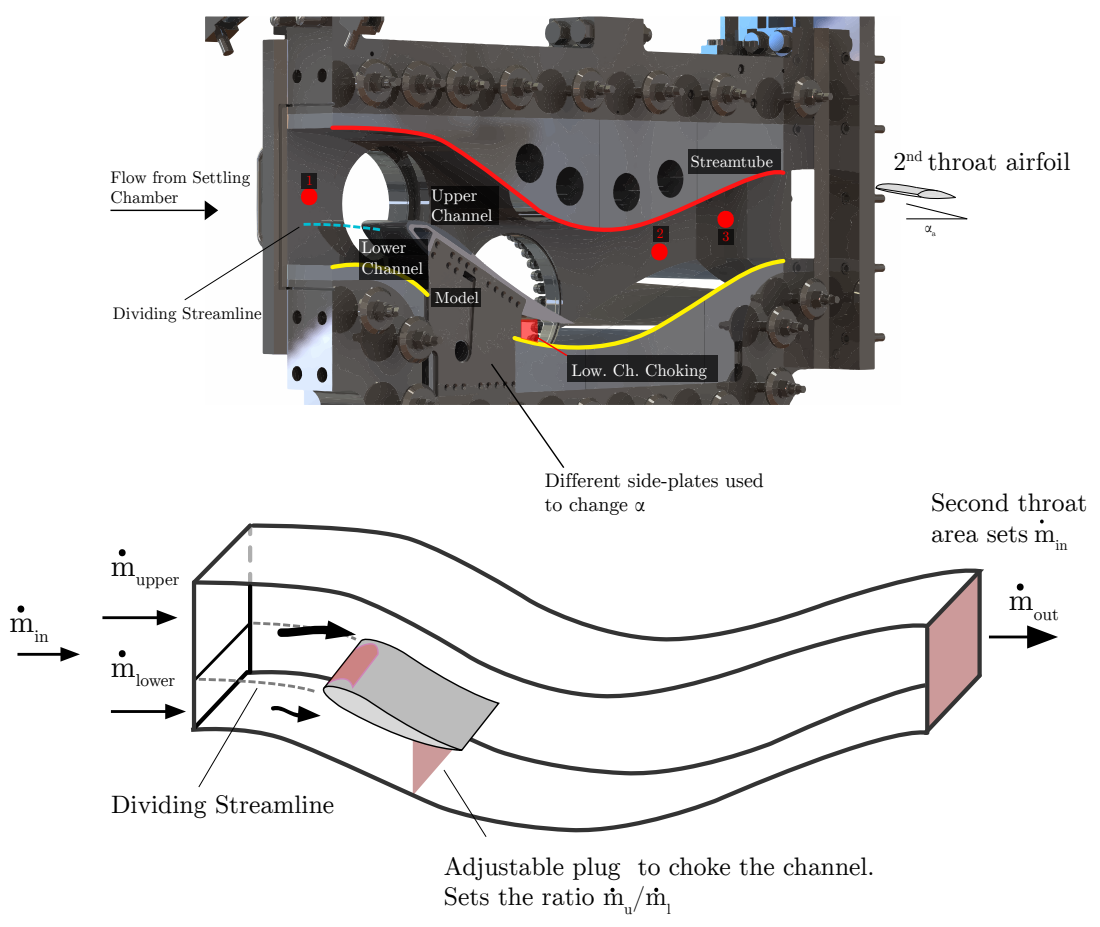

Figure 2. Blow-down wind tunnel working section. Stream-tube design based on computed flow streamlines. Reducing the lower channel area by means of a plug allows an adjustment of the mass flow balance between the upper and lower channel.

The settings listed in Table 1 result in a local flow field closely matching the target flow provided by both experiments and Rolls Royce computational efforts based on an intake travelling at a free-stream Mach number of 0.30 and a representative angle of attack, typical for take-off conditions (Section III.A).

To investigate performance during off-design operation, both angle of incidence and upper channel mass flow rate can be adjusted. By physically changing the model mounting plates, the intake incidence can be varied between 23 and 29 degrees. On the other hand, the mass flow rate into the lower channel is controlled 


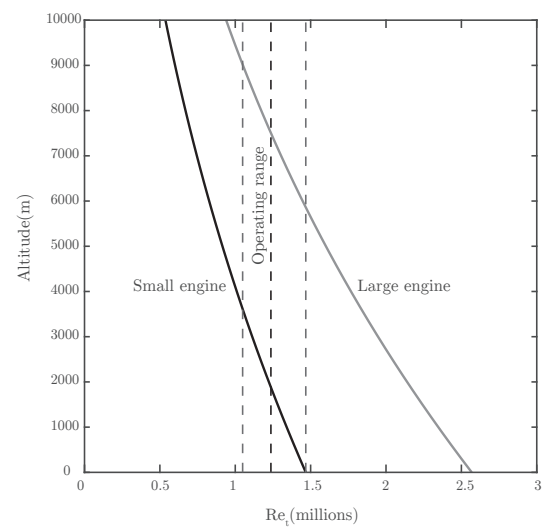

Figure 3. Wind tunnel operating point envelope in terms of entry Reynolds Number.

Table 1. Tunnel entry conditions for on-design investigation

\begin{tabular}{cccccccc}
$\dot{m}(\mathrm{~kg} / \mathrm{s})$ & $M_{\text {entry }}$ & $\alpha(\mathrm{deg})$. & $P_{0}(\mathrm{kPa})$ & $T_{0}(\mathrm{~K})$ & $I_{\%}$ & $\frac{m_{u}}{\dot{m}_{l}}$ & $R e\left(\times 10^{6}\right)$ \\
\hline 8.68 & 0.435 & 23 & 211.6 & $290 \pm 4$ & 1.4 & 3.78 & 1.23 \\
\hline
\end{tabular}

by choking the flow with an adjustable plug, as explained in Figure 2. For a given stagnation pressure, by manually reducing the lower channel area, more mass flow is forced into the upper channel mimicking a greater mass flow demand by the engine. As the ratio between the upper and lower channel mass flows increases, the stagnation streamline is lowered. The effect of the flow resulting from this are presented in Section III C. For on-design investigation, approximately $26.5 \%$ of the overall mass flow rate is passed through the lower channel. This is reduced in subsequent steps to a minimum of $22.5 \%$, which results in a $5.16 \%$ increase in the upper channel mass flow.

A number of different techniques are used to investigate the flow. In particular, a Schlieren technique is used to visualize the features typical of supersonic flows such as shock waves, as well as expansion and compression waves 6 A horizontal knife edge is used and the images were captured at a rate of $4000 \mathrm{fps}$.

Surface pressure measurements are taken during the run to both characterise the flow and to assess experimental repeatability. These measurements are obtained by using pressure taps connected via tubing to a differential pressure transducer. Though small in diameter, the presence of a cavity leads to a complex flow field developing over the tap, resulting in a minor over-prediction of static pressure by approximately $0.5 \%$ at worst for the current configuration $\sqrt[7]{8}$ The accuracy of the transducer is rated at $\pm 0.05 \%$.

Furthermore, a number of these pressure readings are used to calibrate pressure sensitive paint. The photons reflected when the latter is excited by UV light are directly proportional to the pressure acting on it. This is technique particularly suited for moderate to high Mach numbers given the higher pressure gradients involved. The Mach number on the surface considered ranges from $M=0.7$ to $M=1.8$. This is high enough to provide reliable measurements ${ }^{9}$ An optical device is used to measure such luminescence, resulting in a number of pressure readings equivalent to the camera resolution available. The relationship between luminescence intensity and pressure is determined by the Stern-Volmer relation!10

$$
\frac{I_{\text {ref }}}{I_{(P, T)}}=A(T)+B(T) \frac{P}{P_{r e f}}
$$

$I_{\text {ref }}$ is the luminescence taken at a reference pressure and temperature. The obvious choice is to measure the intensity at normal atmospheric conditions with the tunnel off. A second image is taken with the flow on. A no flow - no light background image is subtracted to both images to reduce noise from external light sources. Finally, accordingly to the Stern-Volmer equation, the flow-off reference image is divided by the flow-on image to obtain the pressure ratio. This also helps reducing the impact of thermal effects on the accuracy of PSP. In addition, large temperature changes along the surface are prevented as a result of the 
test model being manufactured with an almost constant thickness, By using the pressure taps located on the model, the values of the constants $A(T)$ and $B(T)$ can be determined and the absolute pressure values can be extracted from the raw light intensity. The overall error associated with pressure sensitive paint can be evaluated by observing the mean deviation of the static pressure measurements from the calibration curve. This is found to be ranging from approximately $2 \%$ to a maximum of $4 \%$. To minimise the calibration error 5-6 different known pressure values are required 10 Given the rig configuration and the position of the access windows, the images are captured at an angle and are, thus, characterised by severe perspective distortion. Therefore, all PSP images have been processed with an un-wrapping algorithm that maps the 3D image onto a two-dimensional surface. In addition to pressure taps, the model is also equipped with four flush-mounted fast-response pressure transducers. Their accuracy is estimated to be $0.5 \%$ of the full scale signal of $100 \mathrm{mV}$.

Flow velocities are measured using a two component Laser Doppler Velocimetry (LDV) system. Two pairs of coherent laser beams, with a wavelength of $561 \mathrm{~nm}$ and $532 \mathrm{~nm}$ respectively, are focused inside the working section to form the interference pattern of the ellipsoidal working volume, measuring $130 \mu \mathrm{m}$ in diameter. Kerosene particles, with a diameter of approximately $0.5 \mu \mathrm{m}, 7$ are used to seed the flow and allow velocity measurements to be recorded via a proprietary software. The laser emitting head and receiving optics are mounted on a traverse capable of moving in one direction with a user defined velocity. The signal is sampled at an optimised variable rate to exploit a full signal cycle leading to a typical measurement accuracy, as stated by the manufacturer, of $\pm 0.1 \%$ of $U_{\max }(\sim 580 \mathrm{~m} / \mathrm{s})$. In addition the emitting head is oriented at an angle $\beta=8.5^{\circ}$ to allow the surface to be reached by the incident beams. A component of the spanwise velocity, $w$, will now affect the measurement of vertical velocity component. The absence of strong span-wise pressure gradients, and the relatively high $v$, due to curved nature of the flow, suggest that $w$ is one order of magnitude lower than $v$. As a consequence of this and of the small angle, the error is expected to be just above $1 \%$. The horizontal velocity component is, on the other hand, unaffected by $\beta$. Proper seeding in the area of interest is crucial to maximise accuracy. The seeding density is consistently high in the free-stream but drops as the wall is approached. As a result, considering the aforementioned sources of error, the overall deviance of the measured values from the real one is estimated to be below $\pm 2 \%$. However, this is higher in the proximity of the wall, within the inner-most portion of he boundary layer. As a consequence, the near wall region is subject to greater uncertainty.

Stagnation temperature is recorded by using 4 T-type thermocouples placed in the settling chamber. A linear increase of stagnation temperature from $\sim 294 \mathrm{~K}$ to $\sim 300 \mathrm{~K}$ is observed during an average 30 second long run. This variation in stagnation temperature is taken into account when converting absolute velocity measurements from LDV to local Mach number to minimise the error involved, which would otherwise peak $1.7 \%$ if a constant stagnation temperature value were assumed.

\section{Results}

\section{A. Baseline flow topology}

The baseline flow field (for conditions, see Table 1) is shown in Figure 4, which depicts both a high speed Schlieren photograph and the Mach number distribution in the interaction region, as measured by LDV. Spatial coordinates are non-dimensionalised by the incoming boundary layer thickness, measured immediately upstream of the pressure rise, to provide grater physical insight. The flow is rapidly turned around the intake highlight (leading edge) and accelerated beyond the local speed of sound via expansions fans created near the front part of the intake. As these intersect the constant pressure boundary at the sonic line, they are reflected as compression waves, which coalesce to form a shock-wave. The interaction between such a shock-wave and the boundary layer, from Schlieren analysis only, appears relatively benign. Laser investigation shows a peak local Mach number of 1.47 in the inviscid flow region upstream of the shock. However, adverse pressure gradients, imposed by the geometry, start decelerating the flow upstream of the shock, resulting in an effective Mach number immediately before the shock foot of 1.4 (Figure 4).

Nonetheless, the pressure gradients imposed by a Mach 1.4 shock wave is generally sufficient to cause shock induced separation. ${ }^{1}$ Recirculation regions may therefore be present but confined to a small region of space. A lambda shock pattern, characteristic of interactions resulting in flow separation, $\frac{11}{11}$ can be inferred from the velocity measurements shown in Figure $4 \mathrm{p}$. Furthermore, the interaction length of $3-4 \delta$ is consistent with shock wave impinging on a slightly separated turbulent SBLI 11 The relatively benign flow despite the severity of the pressure jump imposed by the shock-wave support the hypothesis that the boundary layer might have transitioned to a turbulent state somewhere upstream of the shock foot. 

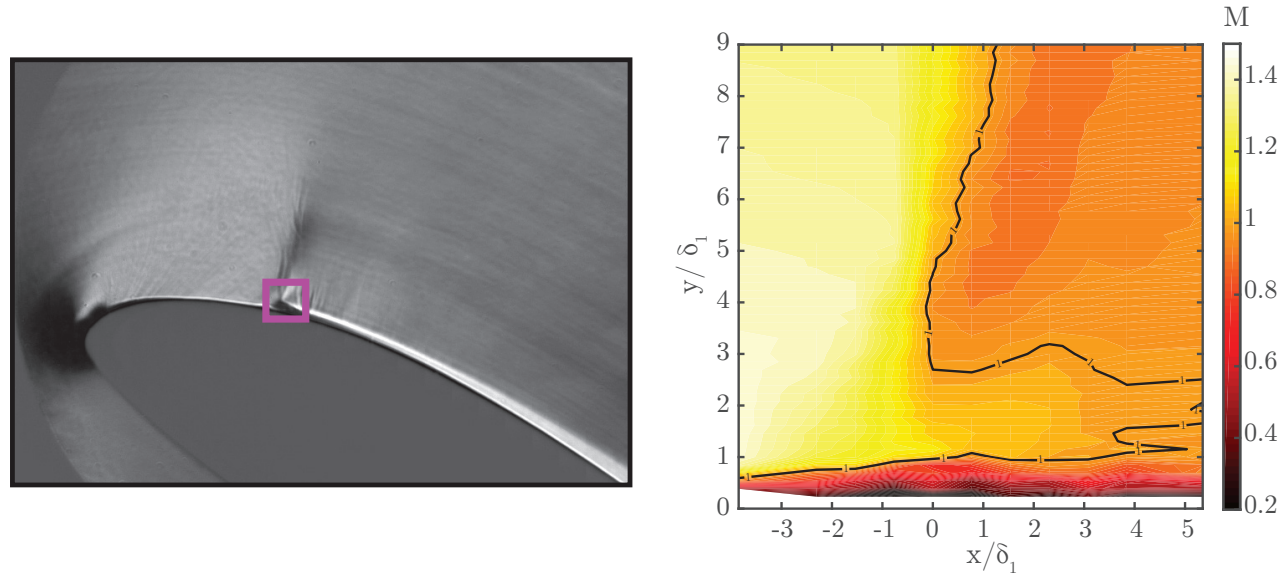

Figure 4. a) Schlieren photograph showing vertical $(d \rho / d y)$ pressure gradients. Flow left to right. b) Mach number contour plot as measured by LDV near the shock - boundary layer interaction region.
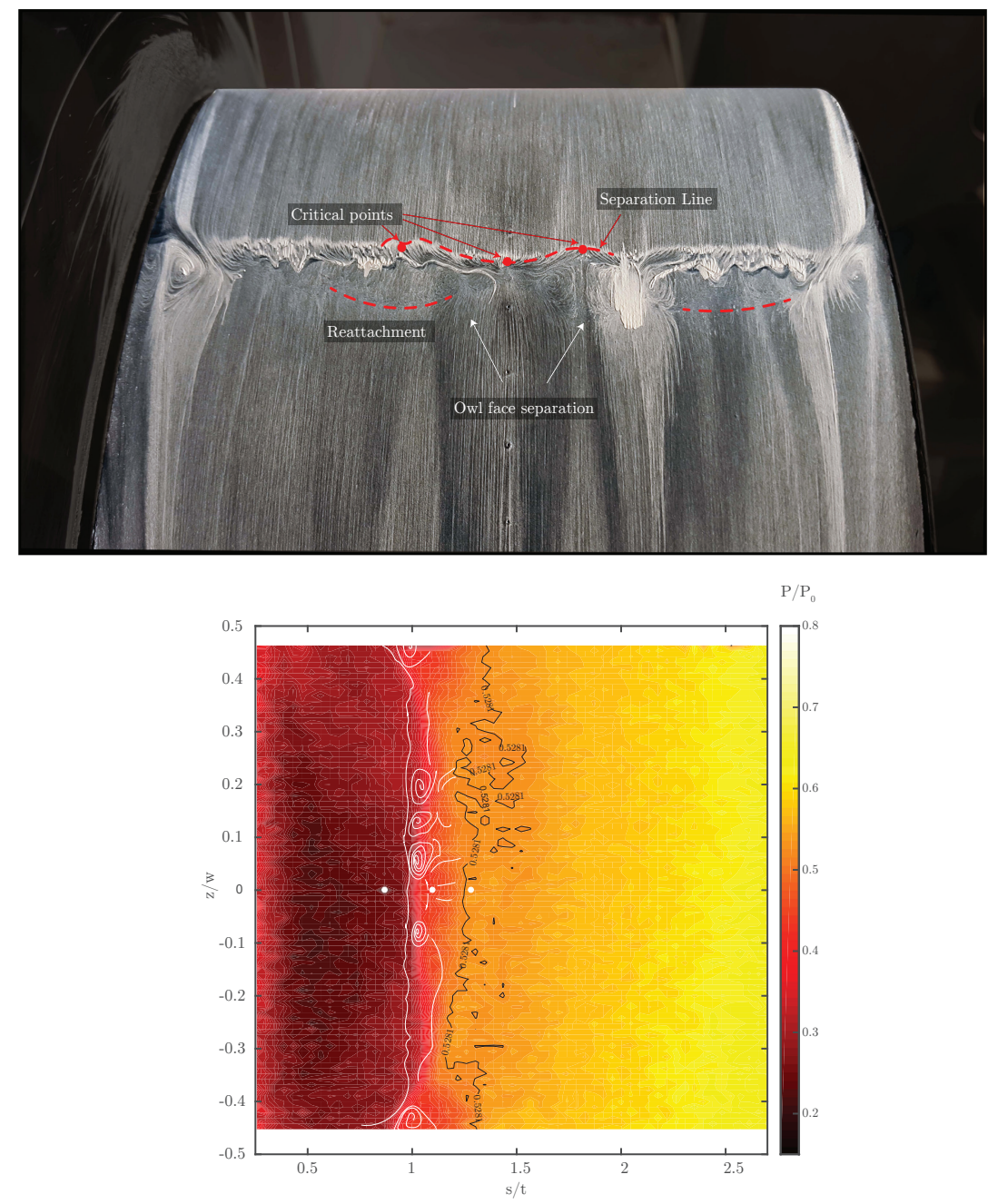

Figure 5. a) Oil flow visualization of the baseline flow. b) Static wall pressure distribution as measured by pressure sensitive paint. Streak-lines extracted by means of adhesive tape. Reattachment occurs before the Mach line highlighted in black. 
Surface oil flow visualization confirms the presence of a three dimensional separation. Figure $5 \mathrm{a}$ depicts a distinct separation line, defined by a number of critical points, namely saddles and nodes. Moreover, three-dimensional bubbles recirculating around their foci can be distinguished downstream of the separation line. The wall pressure along the model surface, evaluated by pressure sensitive paint, is also given in Figure 5. Streak lines, extracted from the oil sediments, have been superimposed to the wall pressure to provide the reader with a more complete depiction of the flow field. The black iso-line indicates $\mathrm{M}=1$, downstream of the interaction. Experience has shown that this line is often located in the proximity of the re-attachment line, shown by the white lines in Figure 5. One explanation for this phenomena is that the secondary supersonic pocket formed downstream of the shock, over the separation bubble, undergoes sudden deceleration at reattachment. Thus, the Mach number drops to subsonic values.

Upon reattachment, the turbulent boundary layer recovers quickly towards an equilibrium state. However, the significant diffusion resulting from the intake geometry imposes considerable adverse pressure gradients onto the subsonic flow. Downstream of the reattachment point, boundary layer measurement were taken at three stream-wise locations to assess the evolution of the near-wall flow. The measurement locations chosen, normalised by lip thickness, are $1.5 t_{m}, 2 t_{m}$ and $2.65 t_{m}$ from the leading edge, with the latter being immediately upstream of a typical fan place. The results are depicted in Figure 6, which shows the normalised boundary layer profiles at the three aforementioned locations. The healthy velocity distributions highlight the fundamentally benign nature of the interaction. At $\mathrm{x}=1.5 t_{m}$, the boundary layer has already recovered to a very full velocity profile, with a shape factor $H_{i} \approx 1.3$. Progressing downstream, the boundary layer thickens and both momentum and displacement thickness increase as a consequence of the adverse pressure gradients imposed by the intake diffuser geometry.
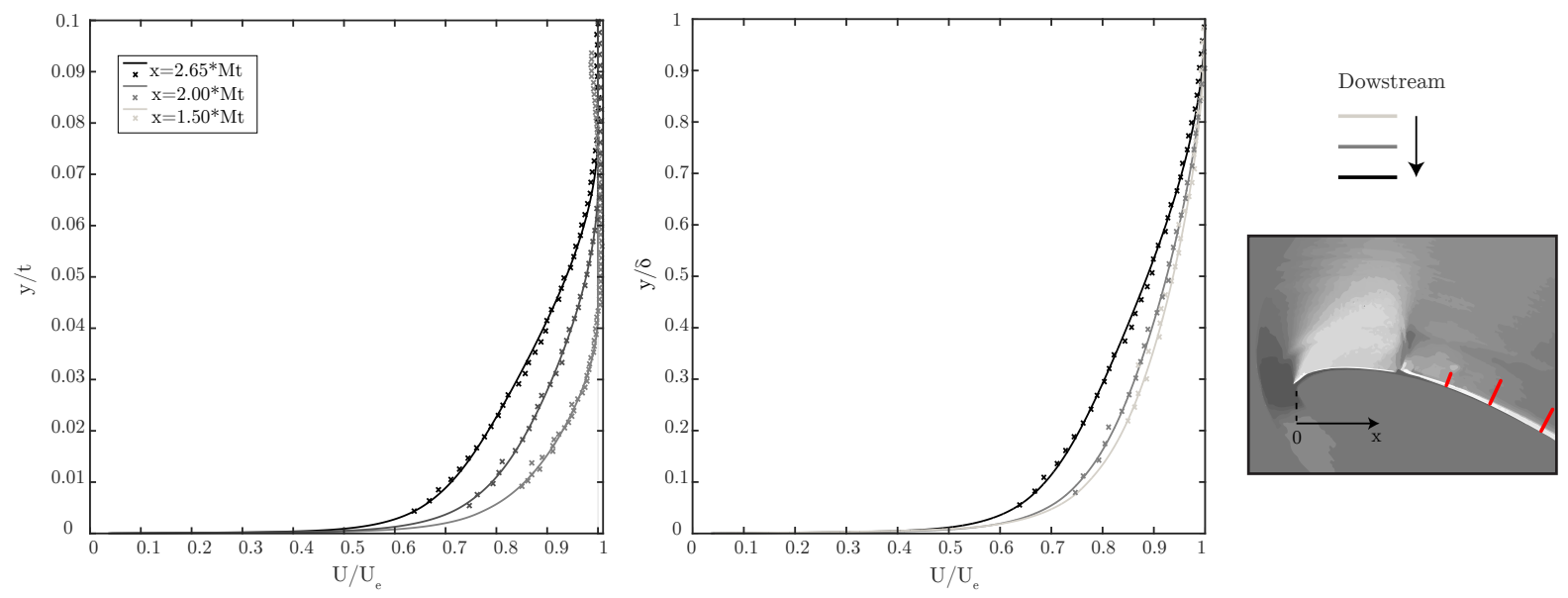

Figure 6. Normalised boundary layer profiles progressing downstream from the interaction. Raw data used to fit a boundary layer profile in order to obtain incompressible integral parameters such as momentum and displacement thickness. Note the effect of adverse pressure gradients on the boundary layer size and fullness.

\section{B. Increasing Incidence}

Two further incidence levels were considered: 25 and 26 degrees respectively. The other global parameters, including mass flow, Reynolds number and mass flow ratio between upper and lower channel are maintained at the values listed in Table 1. In §III.A, the flow topology for the on-design angle of incidence of $23^{\circ}$ was found to be relatively benign, characterised by a moderately strong $\mathrm{M}=1.4$ shock-wave that does not result in largely separated, unsteady, flows. On the contrary, when the operating incidence is increased, onset of flow breakdown is reported. A pronounced $\lambda$ structure is now clearly distinguishable in the Schlieren visualizations presented in both Figure $7 \mathrm{~b}$ and $7 \mathrm{c}$, suggesting a more severe degree of shock-induced separation characterising higher incidence levels. Further increase in angle of incidence, to $26^{\circ}$, did not promote a measurable change in shock position. Nonetheless, the size of the lambda appears larger. Furthermore, as a result of the stronger adverse pressure gradients downstream of the intake throat, the flow breakdown in the diffuser section appears accentuated. In both cases, the flow re-acceleration downstream of the separated interaction causes a moderate supersonic tongue downstream of the normal shock, signified by the presence 
of small secondary shocklets.

Most importantly, the large separation causes a substantial spatial fluctuation of the shock-wave front as a consequence of the inherently unstable nature of recirculating flows. As a result of the unsteady flow, a single Schlieren snapshot is not sufficient to appreciate the shock motion. However, subtracting the time averaged flow-field from each, evenly sampled, Schlieren snapshot and evaluating the root mean square of the light intensity $(\propto d \rho / d y)$, provides a powerful qualitative visualisation of the fluctuations as seen in Figure 8. This suggest that shock wave unsteadiness increases considerably with angle of incidence.

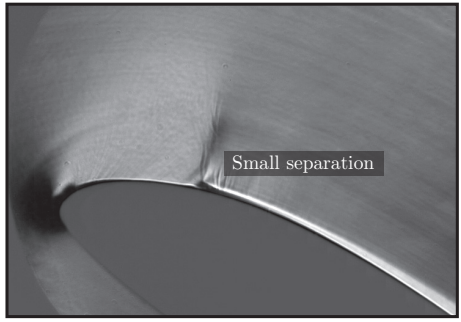

a) Baseline, $23^{\circ}$

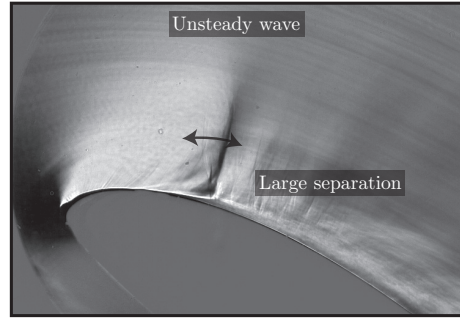

b) $25^{\circ}$

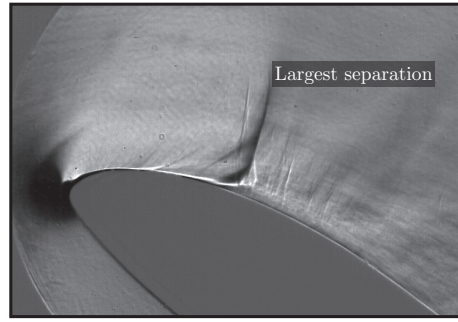

c) $26^{\circ}$

Figure 7. High speed Schlieren photograph comparison for increasing angle of incidence.

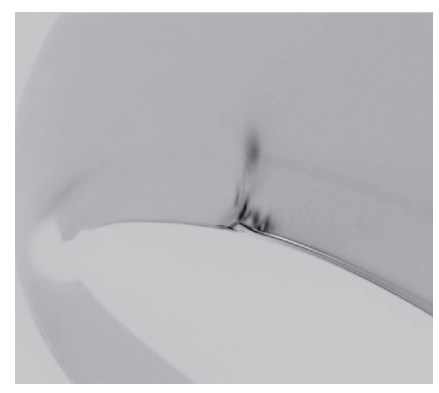

a) $23^{\circ}$

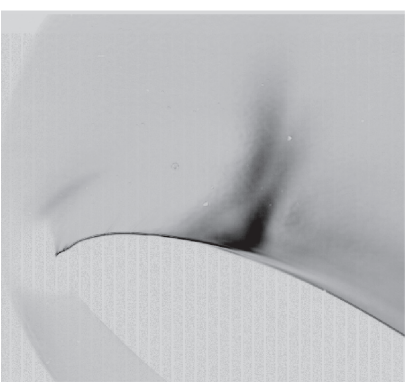

b) $25^{\circ}$

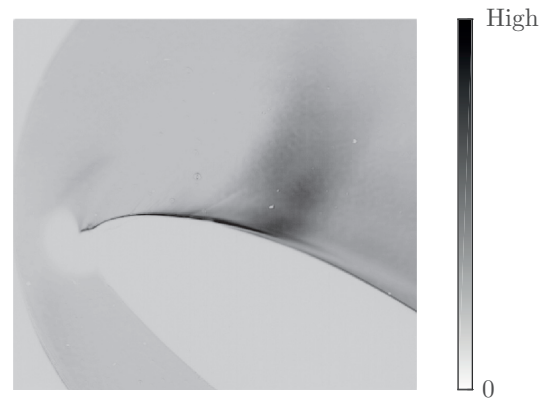

c) $26^{\circ}$

Figure 8. RMS of density gradients obtained from high speed Schlieren photographs sampled at $4 \mathrm{kHz}$.

Due to the largely separated, unsteady, flow at higher incidence, a clear smearing of velocity and pressure measurements can be observed as both involve a degree of time averaging. Nonetheless, they provide valuable insight of the flow-field.

At an incidence of $25^{\circ}$, similar to the baseline flow, there is a small amount of isentropic compression ahead of the shock measured by LDV and distinguishable in the Mach number distribution in Figure 9. However, the peak Mach number ahead of the shock now sits at $\mathrm{M} \approx 1.6$, well above that required to cause flow separation. Further increase in angle of incidence, to $26^{\circ}$, did not result in a measurable change in shock strength, which still sits at $M \approx 1.6$. This is unexpected and might be a sign that the flow is approaching suction peak collapse and intake stall is imminent.

The surface pressure, depicted in Figure 10, also shows increased smearing of the pressure jump with increasing incidence as a result of the larger separation and more important degree of unsteadiness.

Fast response, flush mounted, pressure transducers, located both upstream and downstream of the shockwave, were used to provide further information on the details of the unsteady flow-field.

The transducer signals were sampled at $50 \mathrm{kHz}\left(S t_{c} \approx 50\right)$ and scaled by using the local dynamic pressure.

From the spectra shown in Figure 11, it can be seen that the main shock unsteadiness is found to occur at a Strouhal number, based on chord length and velocity immediately upstream of the shock, of $S t_{c} \approx 0.07$. Such a low frequency oscillation is not surprising if the shock is imagined as a low pass filter, unable to cover large region of space in short time periods (i.e.: high frequency - high amplitude oscillations are unphysical) ${ }^{12}$ The energy content rapidly approaches zero at higher frequencies. However, significant broad 


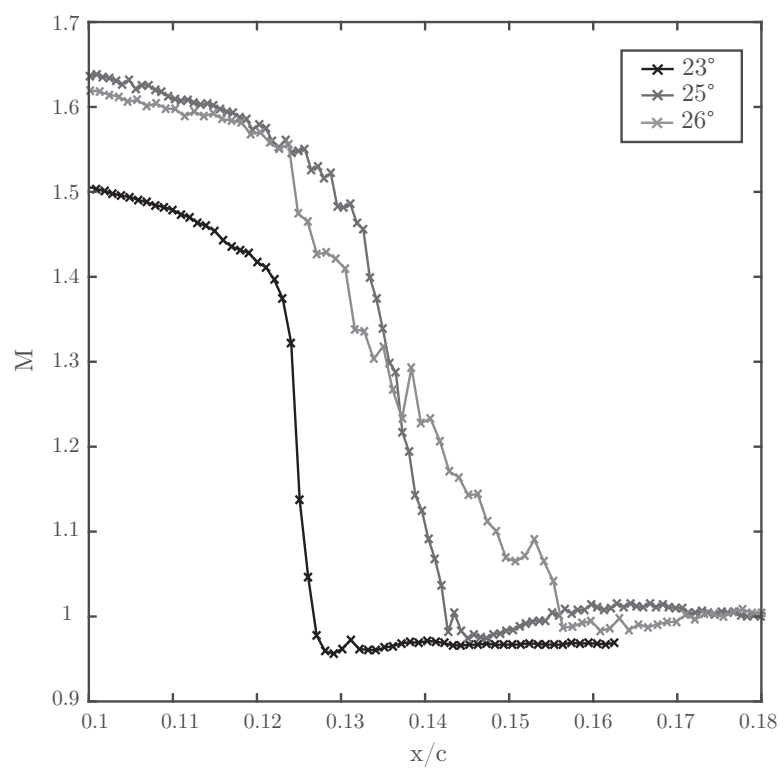

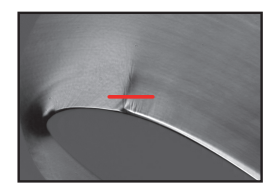

a) $23^{\circ}$

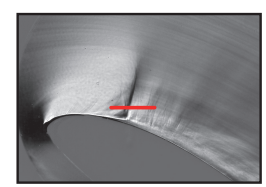

b) $25^{\circ}$

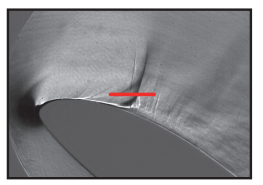

c) $26^{\circ}$

Figure 9. Stream-wise traverse across the inviscid region of the shock wave at the locations indicated by the red line. Higher incidence results in greater acceleration upstream of the interaction and consequently stronger shock wave.
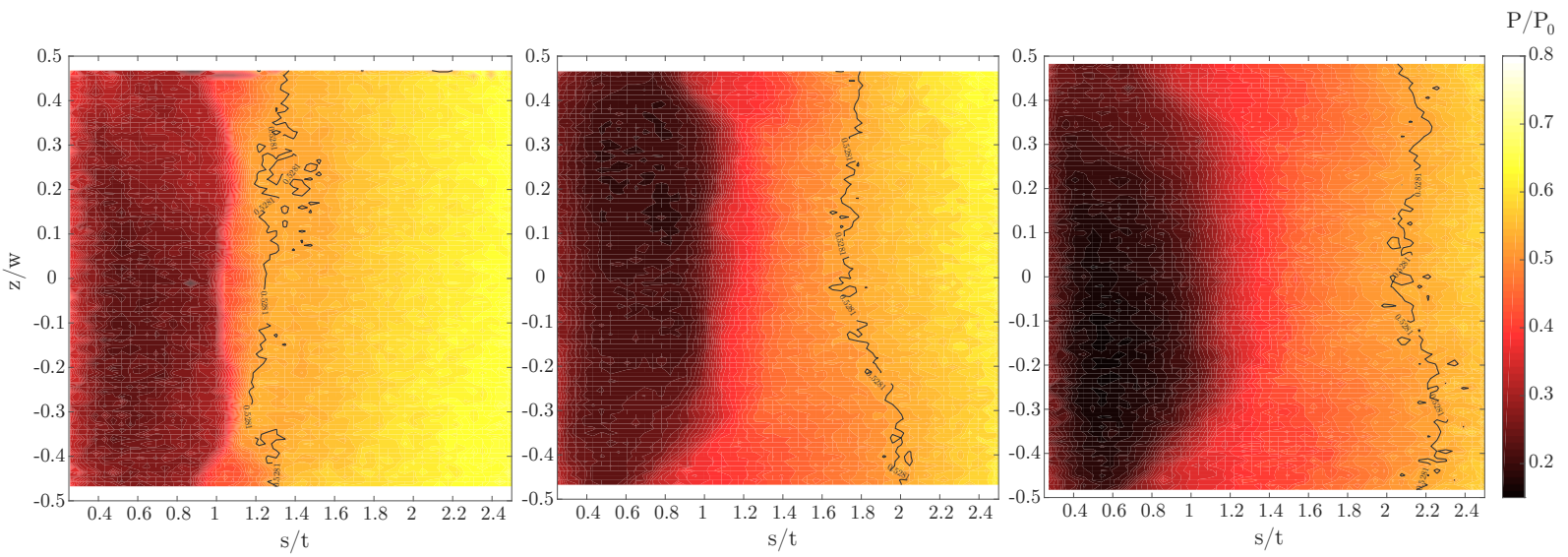

Figure 10. Static wall pressure along the surface of the intake for increasing incidence. Extracted from pressure sensitive paint.

noise band is present between $1<S t_{c}<3$. Empty tunnel measurements suggest this to be a tunnel artefact.

At the present time, the origin of the disturbance causing the shock oscillation is still unclear. The measured period of oscillation is consistent with an acoustic wave propagating upstream from the trailing edge at a speed of $a-u$ (where $a$ is the local speed of sound and $u$ flow velocity) and, upon reaching the shock-front, the disturbance is convected back towards the trailing edge with the flow. However, the cross correlation of the pressure signals suggests considerably lower downstream convection speeds of the order of $\approx 10 \%$ of the free-stream velocity. As a result, investigation is still ongoing. Crucially, however, it is noted that low frequency oscillation of the shock wave is reported only when significant separated flow is present. For the lowest incidence considered, no shock oscillation is seen.

Wall normal velocity measurements were taken at a stream-wise location $x=2.65 t_{m}$, close to the turbofan plane. The resulting boundary layer profiles are given, in both physical size and normalised by their thickness, in Figure 12.

It can be seen that the boundary layer grows quickly for relatively small increases in incidence. Further- 

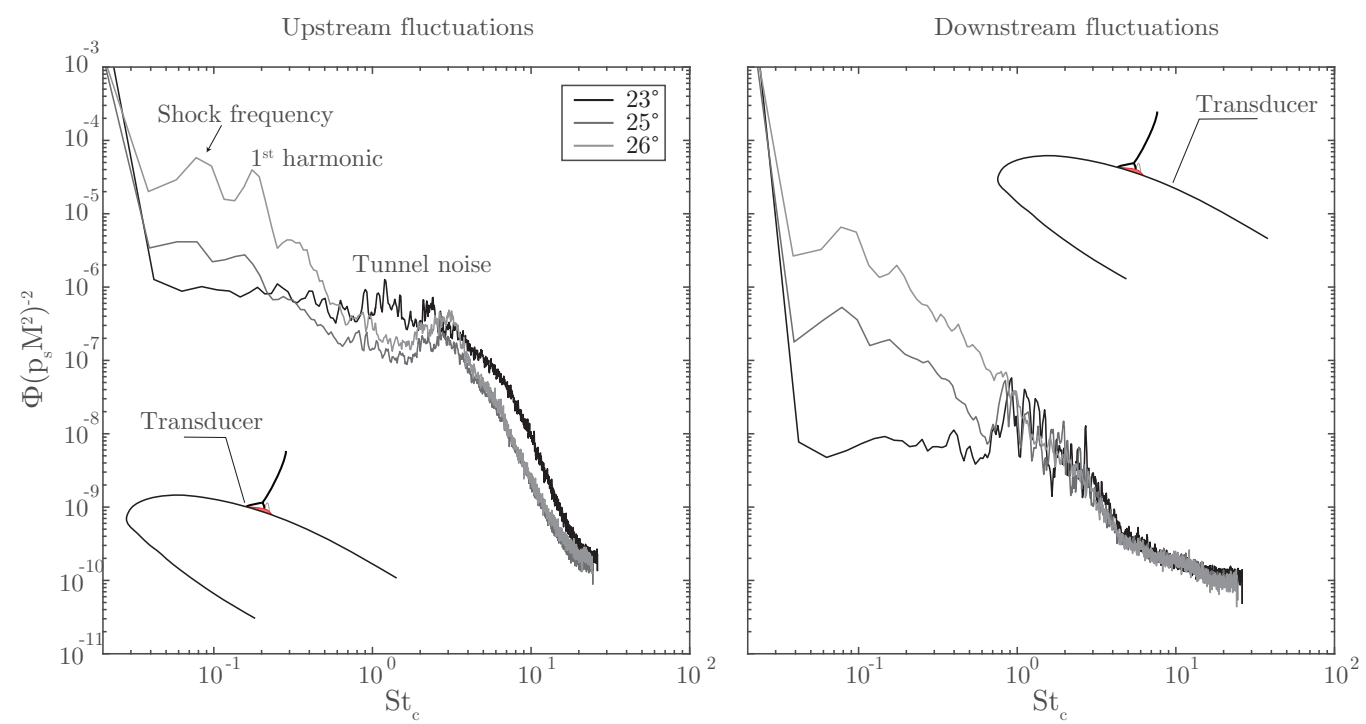

Figure 11. Power spectral density of the pressure fluctuations upstream and downstream of the shock front respectively. Fluctuations measured by fast response traducers normalised by local dynamic pressure.
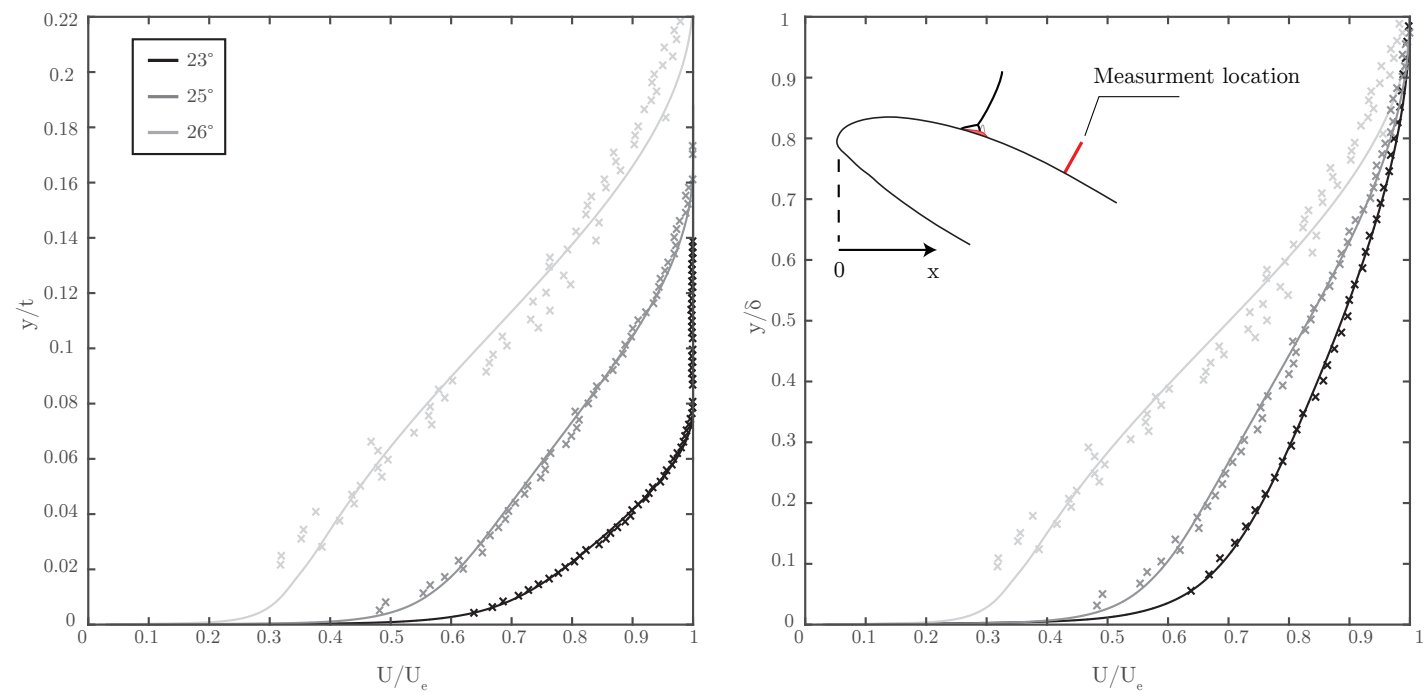

Figure 12. Wall normal velocity measurements downstream of the interaction. Higher incidence shows a thicker viscous layer and noticeably less full profile in the outer region due to increased wave drag.

more, the velocity profile is also adversely affected by the more severe pressure gradients with the largest angle of attack being considerably less full than the baseline and a rapid rise of incompressible shape factor as incidence increases is reported. Incompressible integral parameters are listed in Table 2, As easily inferred from Figure 12, both displacement thickness $\delta_{i}^{*}$ and momentum thickness $\theta$ grow alongside the boundary layer physical size $\delta$ as incidence is increased. The baseline case is typical for an equilibrium turbulent profile, whereas the highest incidence case shows a rather unhealthy shape factor. This suggest that reattachment might have occurred a short distance upstream.

\section{Increasing "Engine Demand"}

So far, only a fixed mass flow ratio between upper and lower channel of the working section has been considered. However, the operational envelope of an engine intake includes a further variable, i.e.: the 
Table 2. Incompressible boundary layer parameters for increasing incidence

\begin{tabular}{ccccc}
$\alpha$ (deg.) & $\delta_{i} / t$ & $\delta_{i}^{*} / t$ & $\theta_{i} / t$ & $H_{i}$ \\
\hline 23 & 0.078 & 0.011 & 0.008 & 1.345 \\
25 & 0.184 & 0.037 & 0.025 & 1.483 \\
26 & 0.225 & 0.072 & 0.036 & 1.971 \\
\hline
\end{tabular}

engine mass flow demand. For the baseline configuration $\approx 75 \% \dot{m}$ is discharged via the upper channel. The following section examines the effect of increasing this value by $\approx 5 \%$ in three, equally spaced steps. A first, qualitative, depiction of the flow field is given in Figure 13, showing Schlieren photographs for four different upper channel mass flow values. These images clearly indicate that the three incidence levels respond differently to changes in mass flow ratio.

For the more benign $23^{\circ}$ case, as the mass flow in the upper channel increases, the shock wave moves further downstream and the supersonic region grows significantly. The LDV measurements in Figure 14 show a greater degree of isentropic compression ahead of the shock as this moves downstream. To some extent, this counteracts the larger acceleration. Nonetheless, the Mach number just ahead of the shock shows an increase with upper channel mass flow from $\mathrm{M}=1.4$ for the baseline to $\mathrm{M}=1.5$ for the largest mass flow ratio considered. With increasing shock strength the interaction domain grows and eventually a clear $\lambda$ structure emerges indicating flow separation (see Figure 13). The size of the lambda, even for the greatest mass flow considered, is, however, relatively small, suggesting that the separation remains of modest extent.
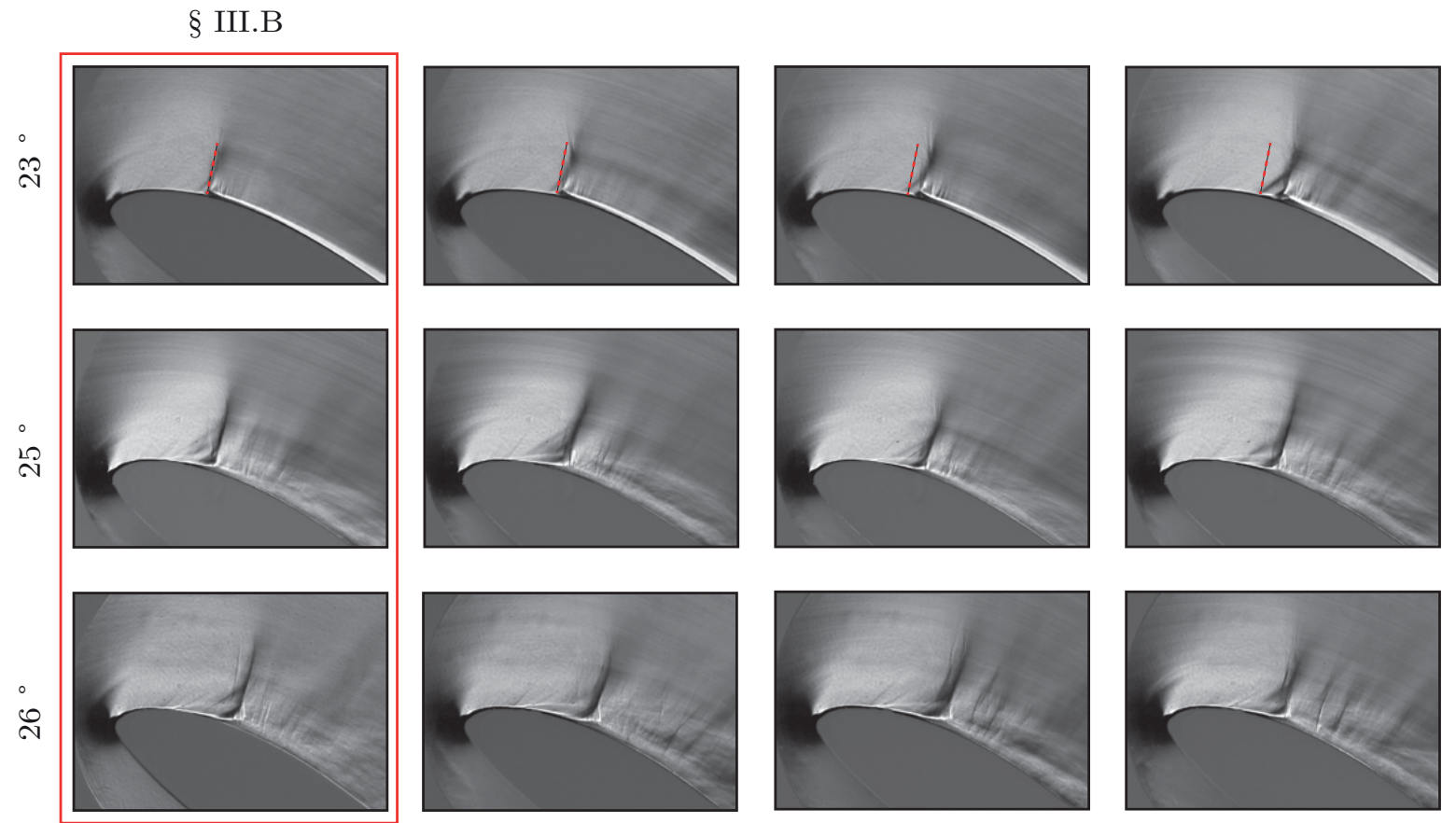

$+1.72 \dot{\mathrm{m}}_{\mathrm{u}}$

$+3.44 \dot{\mathrm{m}}_{\mathrm{u}}$

$+5.16 \dot{\mathrm{m}}_{\mathrm{u}}$

Figure 13. Schlieren photographs captured by means of a high-speed camera for the three angle of incidence. Note very little change for the higher incidence levels.

For the more severe interactions occurring at higher incidence, however, both qualitative and quantitative measurements suggest a reduced sensitivity to mass flow rate increases. The already unsteady and separated flow characterising the $25^{\circ}$ and $26^{\circ}$ cases shows a less pronounced response to mild variations of the stagnation 
point location. LDV measurements across the shock wave, shown in Figure 14b-c, demonstrate a considerably smaller increase in upstream Mach number. Also, there is no significant change in shock location at these higher incidences. This is likely to be due to the largely separated flow downstream that prevents the shock from moving further into the diffuser.
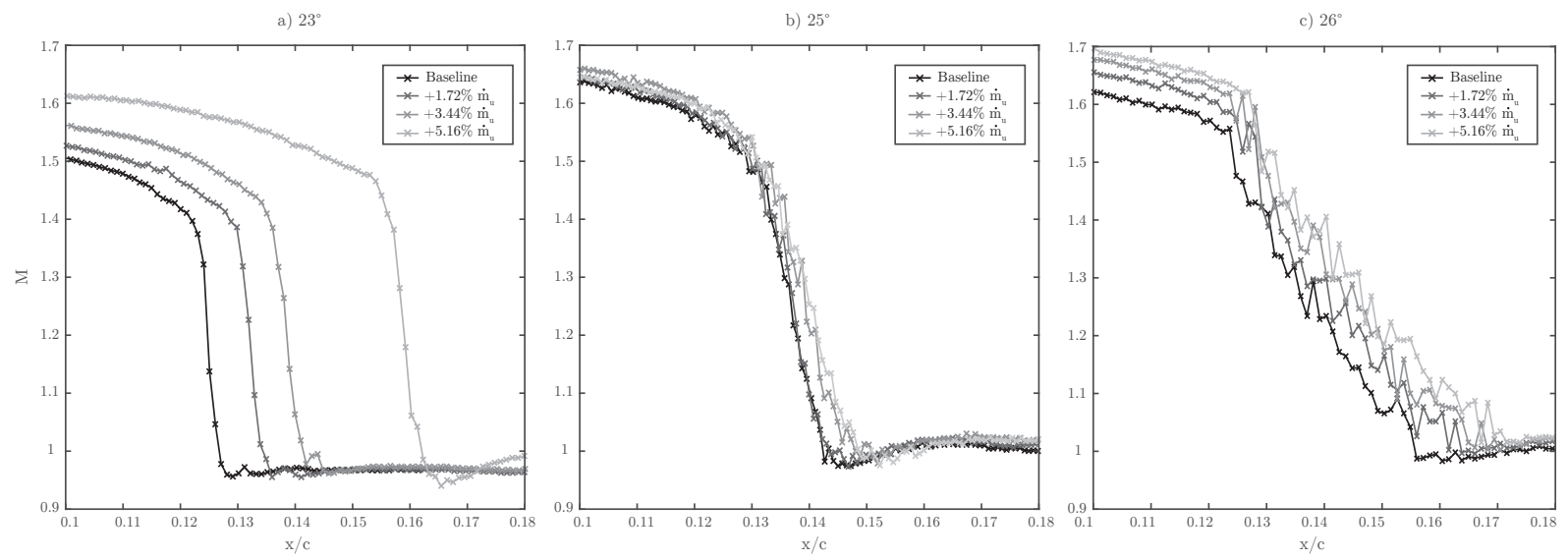

Figure 14. Series of stream-wise velocity measurements across the inviscid region of the shock-wave for the two incidence configurations across the range of upper channel mass flows.

These observations are further confirmed by surface pressure distributions (from PSP) in Figure 15. The pronounced downstream motion of the shock for the lowest incidence case is captured clearly by the pressure field. The Mach line moves further from the main shock, suggesting an increase in shock induced separation. This was also signified by the presence of a more pronounced lambda-shock pattern in the Schlieren images (Figure 13) and is a consequence of the increased shock strength. Consistent with velocity measurements, the changes observed for the higher incidence cases are less dramatic as the shock position remain practically unchanged. Furthermore, the approximate extent of separation (as estimated from the location of the Mach line) remains almost constant.

Figure 16 summarises the changes in integral boundary layer properties, measured at an axial location $x=2.65 t_{m}$, with upper channel mass flow rate. It should be noted that increasing $\frac{m_{u}}{\dot{m}_{l}}$ results in a stagnation point shifted downstream along the lower surface. Thus, it has a similar effect to an increase in effective angle of incidence.

Increases in the growing length cause the low momentum flow region near the wall to more susceptible to such changes as every incidence considered shows the same trend of a measurably thicker boundary layer near the virtual engine face at $x=2.65 t_{m}$.

The integral parameters, in Figure 16, highlight once again the difference in the flow response to changes in mass flow at different incidence levels. At the lowest incidence the measurements reveal a somewhat unexpected result. As the shock gets stronger and moves downstream, closer to the measurement plane at $x=2.65 t_{m}$, the boundary layer appears to gain fullness, with a measurable drop in shape factor $H$. Similar effects are also seen in the boundary layer velocity distribution shown in Figure 17, where the $23^{\circ}$ case shows a slightly improvement in the 'best fit' theoretical profile as mass flow rate is increased. This implies that not only has the separated boundary layer recovered faster from an increasingly stronger shock-wave, but also that it recovers to a marginally healthier velocity profile. Displacement and momentum thickness show a similar, albeit less pronounced, downward trend except for the largest $\dot{m}_{u}$ case. This could be explained as follows: shape factor increases considerably at separation. At reattachment, this drops very quickly to equilibrium values. However, shape factor will start increasing downstream due the adverse pressure gradients imposed by the geometry (see Figure 6). Thus, there will be a point at which shape factor reaches its minimum value. As the shock moves downstream with mass flow rate increase, so does this point. Since the measurements shown in Figure 16 and 17. the smaller shape factor might be a consequence of this minimum point moving closer to the measurement plane.

In contrast, for greater incidence angles, where no change in shock position is reported, this trend is not observed and the boundary layer state deteriorates with mass flow rate. However, the reported changes in integral parameters are small and can be deemed to be within experimental error. 

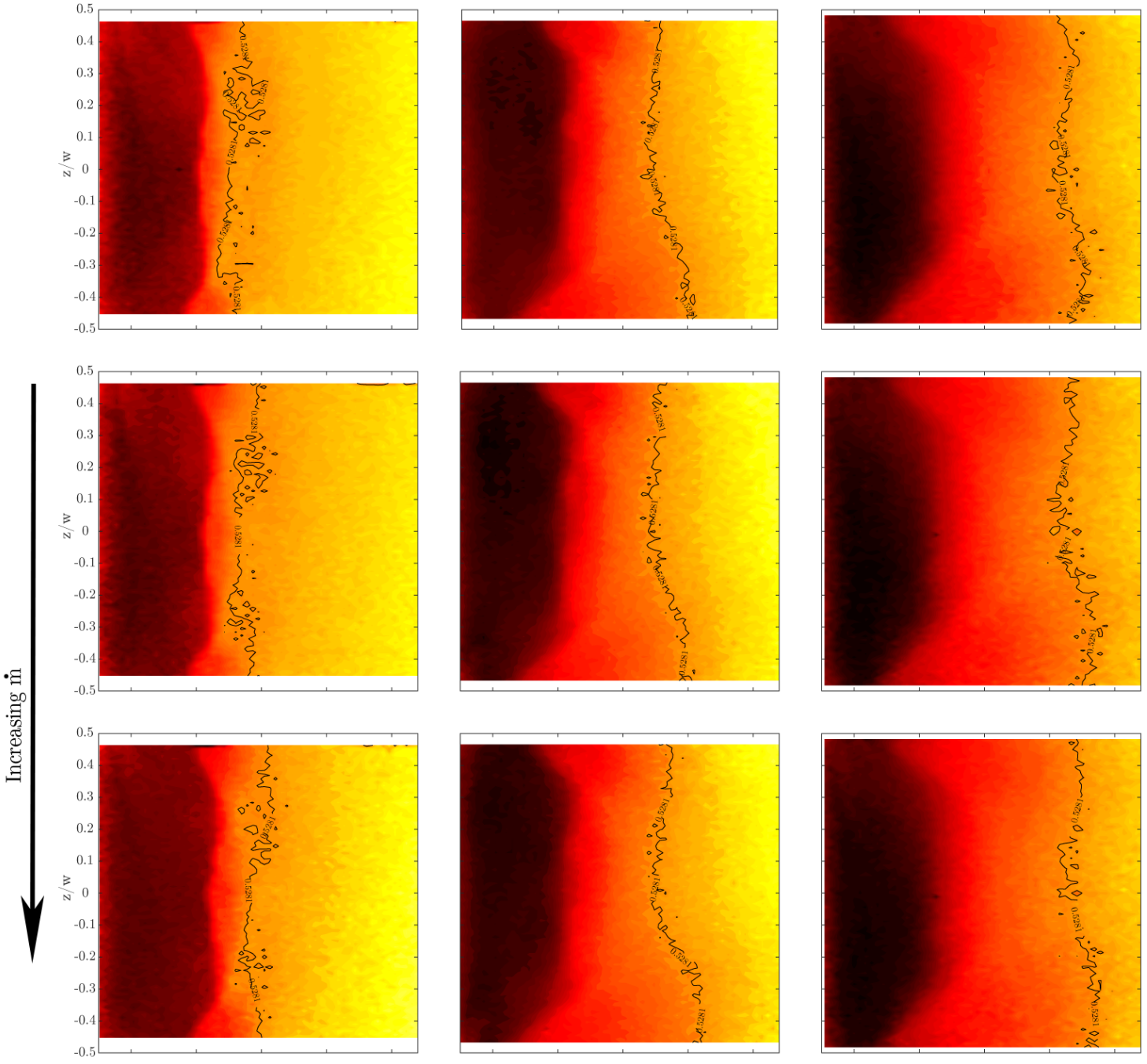

$\mathrm{P} / \mathrm{P}_{0}$
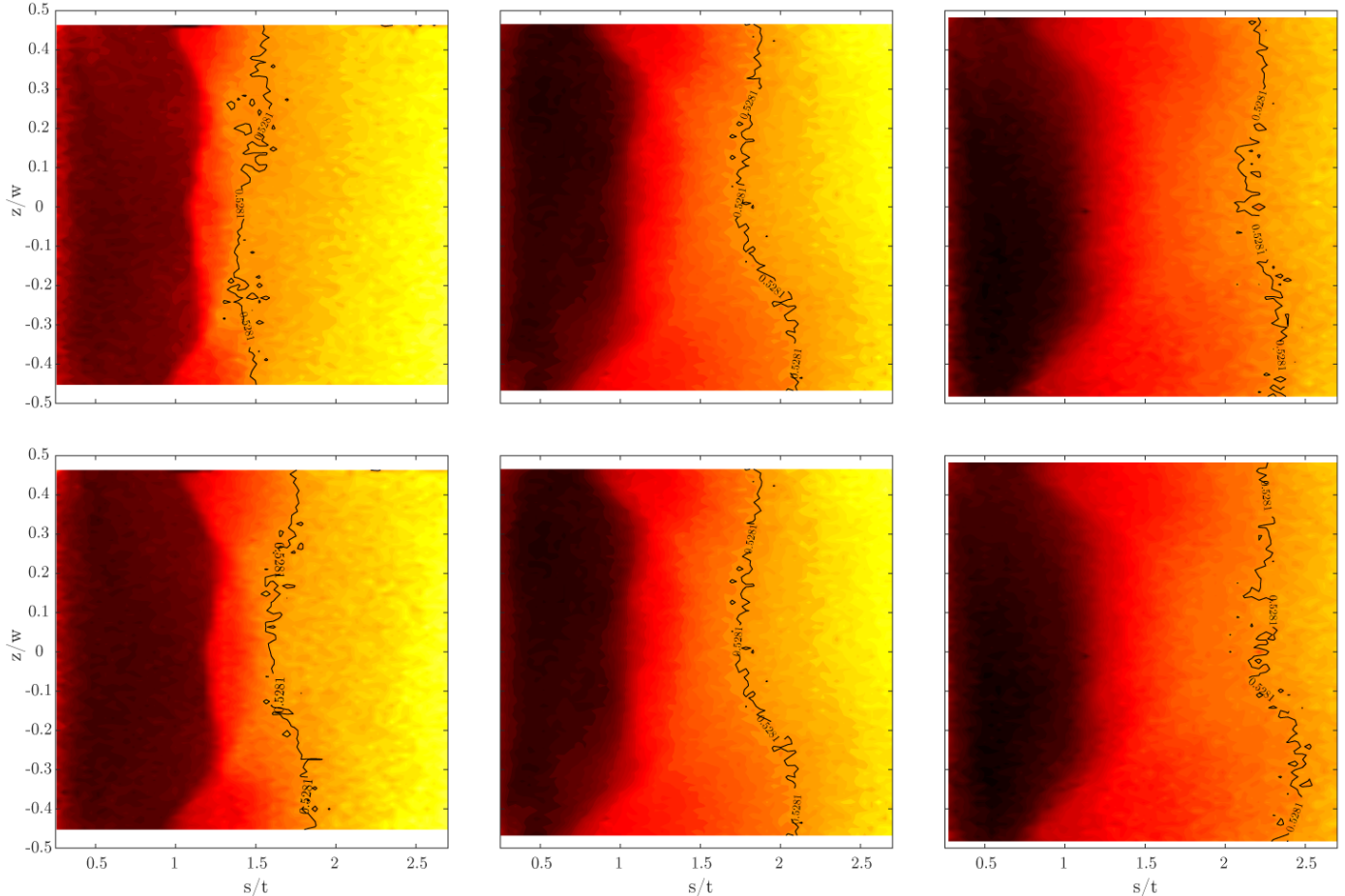

Figure 15. Static wall normal pressure as measured by PSP and processed via a flattening algorithm. Increased incidence show a reduced sensitivity to mass flow ratio increases. Whereas the shock position and strength is noticeably different for the 23 degrees, the changes are minimal for the higher incidence cases. Moreover, the length of separation, estimated by using the position of the Mach line, does not show important changes. 

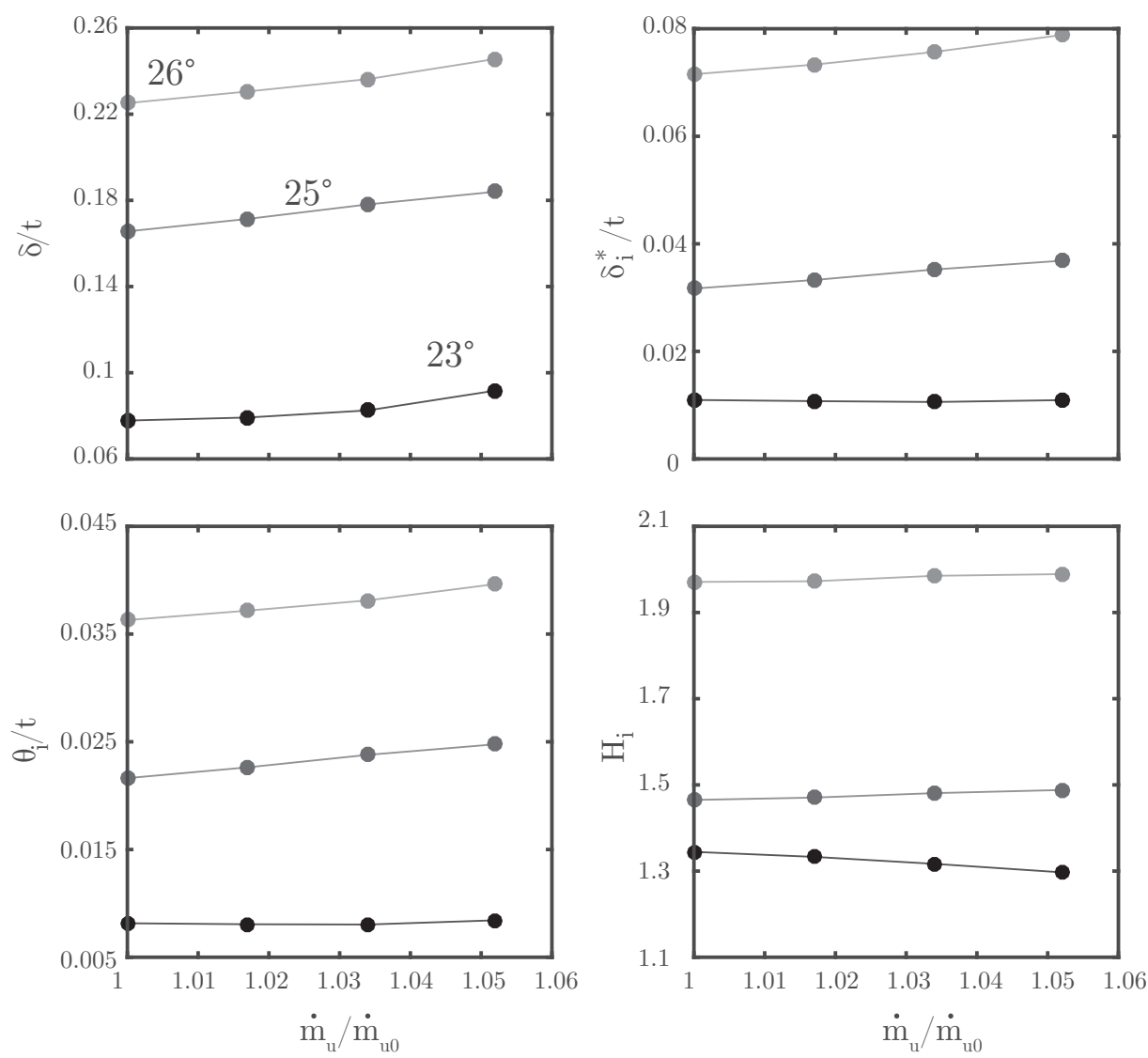

Figure 16. Comparison between boundary layer properties at the three incidence levels considered for increasing upper channel mass flow.
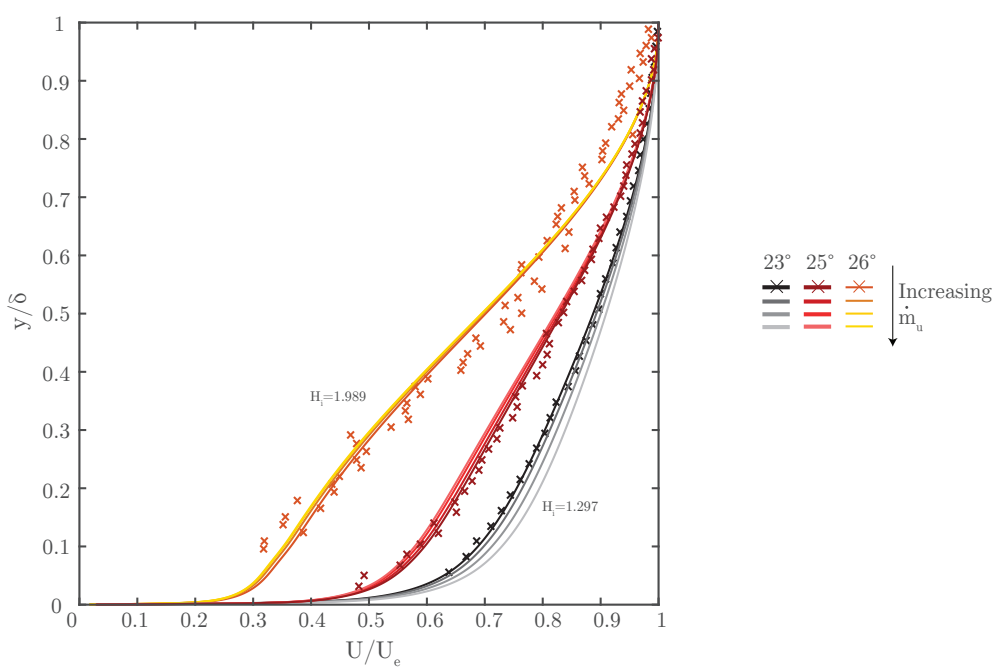

Figure 17. Variation of boundary layer parameters for increasing angle of incidence with increasingly large upper channel mass flow. Data recoded normal to the wall surface a the most downstream position $\left(x=2.65 t_{m}\right)$. 


\section{Conclusion}

A novel rig has been used to investigate the shock-wave boundary layer interaction occurring over the lower lip of transonic engine intakes at incidence. A particular focus of this study is the formation of shock waves downstream of the highlight and the development of separation with increasing incidence and engine mass flow rates. During typical on-design conditions, such as normal take-off, the flow field is found to be relatively benign, consisting of a $\approx$ Mach 1.4 normal shock forming upstream of the throat plane and only a small degree of shock-induced separation was reported.

This changes as angle of incidence is increased. Three incidence levels have been studied: the reference angle of $23^{\circ}$, and two more severe configurations, at $25^{\circ}$ and $26^{\circ}$ respectively. At these incidence levels, the departure from the baseline reference flow is significant, with even a modest $2^{\circ}$ increase resulting in a noticeably greater degree of shock-induced separation, ultimately leading to the onset of moderate to severe unsteadiness. Further increase in angle of incidence, resulted in even greater separation and larger shock oscillation amplitude. For both cases, the oscillation frequency is at the low end of the spectrum $(S t \approx 0.08)$. On the other hand, increasing 'engine demand' has contradictory effects at different incidence levels. For the lowest angle considered $\left(23^{\circ}\right)$, as the flow through the upper channel increases, the shock moves proportionally downstream and its strength grows. For larger angle of incidence, on the other hand, there is a reduced sensitivity to mass flow rates. In fact, no major growth in unsteadiness amplitude, nor shift of the mean shock location, were observed. This is unexpected but may be a sign that the flow is approaching full breakdown and potentially suction peak collapse. Nonetheless, the evidence collected so far suggests that increases in angle of incidence have a greater effect on the growth of separation and onset of flow unsteadiness than increases in engine mass flow rate.

\section{Acknowledgements}

The authors wish to acknowledge Dave Martin, Sam Flint, Anthony Luckett and John Hazlewood for operating the CUED blow-down wind tunnel. Moreover, they would like to thank Rolls Royce Plc, the Engineering and Physical Sciences Research Council (EPSRC) and the National Wind Tunnel Facility for invaluable (NWTF) contribution towards this research.

\section{References}

${ }^{1}$ BABINSKY, H. and HARVEY, J. K., Shock-wave-Boundary layer Interactions, Cambridge University Press, 2011.

${ }^{2}$ JAKUBOWKI, A. K., "Internal cowl-separation at high incidence angles," 13th Aerospace Sciences Meeting. Pasadena,CA,USA., 1975.

${ }^{3}$ CHOU, D. C., LUIDENS, R. W., and STOCKMAN, N. O., "Prediction of Boundary-Layer Flow Separation in V/STOL Engine Inlets," Journal of Aircraft, Vol. 15, No. 8, 1978.

${ }^{4}$ COSCHIGNANO, A. and BABINSKY, "Onset of unsteadiness in aero-engine intakes at incidence," 55th AIAA Aerospace Sciences Meeting, AIAA SciTech Forum, (AIAA 2017-1435), 2017.

${ }^{5}$ MAKUNI, T. E., BABINSKY, H., SLABY, M., and SHEAF, C. T., "Shock Wave-Boundary-Layer Interactions in Subsonic Intakes at High Incidence," 53rd AIAA Aerospace Sciences Meeting. January, 2015.

${ }^{6}$ FERRI, A., Elements of Aerodynamics of Supersonic Flows, Macmillan Co., 1949.

${ }^{7}$ COLLISS, S. P., Vortical structures on three-dimensional shock control bumps, PhD Thesis, University of Cambridge, 2014.

${ }^{8}$ MEIER, H., "Measuring techniques for compressible turbulent boundary layers," NASA STI/Recon Technical Report N, Vol. 79, Nov. 1977.

${ }^{9}$ Sajben, M., "Uncertainty estimates for pressure sensitive paint measurements," AIAA Journal, Vol. 31, No. 11, 1993, pp. 2105-2110.

${ }^{10}$ GREGORY, J. W., ASAI, K., KAMEDA, M., LIU, T., and SULLIVAN, J. P., "A review of pressure-sensitive paint for high-speed and unsteady aerodynamics," Proc. IMechE Vol. 222 Part G: J. Aerospace Engineering, 2007.

${ }^{11}$ DÉLERY, J. M., MARVIN, J. G., and RESHOTKO, E., "Shock-Wave Boundary Layer Interactions," AGARDograph, Vol. 280, March 1986.

${ }^{12}$ Dussauge, J.-P., Dupont, P., and Debiève, J.-F., "Unsteadiness in shock wave boundary layer interactions with separation," Aerospace Science and Technology, Vol. 10, No. January, 2006, pp. 85-91. 